\title{
Shear stress-induced changes in atherosclerotic plaque composition are modulated by chemokines
}

\author{
Caroline Cheng, ${ }^{1}$ Dennie Tempel, ${ }^{1}$ Rien van Haperen, ${ }^{2}$ Hetty C. de Boer, ${ }^{3}$ Dolf Segers, ${ }^{1}$ \\ Martin Huisman, ${ }^{1}$ Anton Jan van Zonneveld, ${ }^{3}$ Pieter J.M. Leenen, ${ }^{4}$ \\ Anton van der Steen, ${ }^{1}$ Patrick W. Serruys, ${ }^{1}$ Rini de Crom, ${ }^{2,5}$ and Rob Krams ${ }^{1,6}$
}

\begin{abstract}
1Department of Cardiology, Thoraxcenter, and 'Department of Cell Biology, Erasmus University Medical Center, Rotterdam, The Netherlands. ${ }^{3}$ Department of Nephrology, Leiden University Medical Center, Leiden, The Netherlands. ${ }^{4}$ Department of Immunology and ${ }^{5}$ Department of Vascular Surgery, Erasmus University Medical Center, Rotterdam, The Netherlands. ${ }^{6}$ Department of Physiology, Vrije Universiteit Amsterdam, Amsterdam, The Netherlands.
\end{abstract}

\begin{abstract}
We previously found that low shear stress (LSS) induces atherosclerotic plaques in mice with increased lipid and matrix metalloproteinase content and decreased vascular smooth muscle and collagen content. Here, we evaluated the role of chemokines in this process, using an extravascular device inducing regions of LSS, high shear stress, and oscillatory shear stress (OSS) in the carotid artery. One week of shear stress alterations induced expression of IFN- $\gamma$-inducible protein-10 (IP-10) exclusively in the LSS region, whereas monocyte chemoattractant protein-1 (MCP-1) and the mouse homolog of growth-regulated oncogene $\alpha$ (GRO- $\alpha$ ) were equally upregulated in both LSS and OSS regions. After 3 weeks, GRO- $\alpha$ and IP-10 were specifically upregulated in LSS regions. After 9 weeks, lesions with thinner fibrous caps and larger necrotic cores were found in the LSS region compared with the OSS region. Equal levels of MCP-1 expression were observed in both regions, while expression of fractalkine was found in the LSS region only. Blockage of fractalkine inhibited plaque growth and resulted in striking differences in plaque composition in the LSS region. We conclude that LSS or OSS triggers expression of chemokines involved in atherogenesis. Fractalkine upregulation is critically important for the composition of LSS-induced atherosclerotic lesions.
\end{abstract}

\section{Introduction}

Shear stress, which is the drag force per unit area acting on the endothelium as a result of blood flow, plays a critical role in plaque location and progression $(1,2)$. Both low shear stress (LSS) and oscillatory shear stress (OSS) have been implicated as proatherogenic in observational studies in humans and animals $(1,3,4)$. Using a newly developed method to study cause-effect relationships of shear stress in vivo (5), we were able to demonstrate that LSS and OSS are both important stimuli for the induction of atherosclerosis (6). Remarkably, LSS induces the development of larger-sized lesions with decreased lipid content and MMP activity and increased numbers of VSMCs and collagen content compared with OSS-triggered lesions.

Several studies have indicated the role of inflammatory cells in the pathogenesis of plaques with a vulnerable phenotype (7-11). Furthermore, it has been postulated that rupture of vulnerable plaques is associated with activation of macrophages and of the adaptive immune system, leading to metalloproteinase activity and collagen breakdown $(9,12,13)$. Accumulation of inflammatory cells occurs through a series of events consisting of rolling, arrest, and migration $(14,15)$. Capture and initial rolling are induced by the interaction of several proteins, while arrest occurs predominantly by integrin-

Nonstandard abbreviations used: BCA, brachiocephalic artery; CCL14, C-C chemokine ligand 14; CX3CR1, C-X3-C chemokine receptor 1; ELC, EBI1 ligand chemokine; GRO- $\alpha$, growth-regulated oncogene $\alpha$; IP-10, IFN- $\gamma$-inducible protein-10; LSS, low shear stress; MCP-1, monocyte chemoattractant protein-1; NAP-2, neutrophil-activating protein-2; OSS, oscillatory shear stress; QPCR, quantitative real-time PCR; SDF-1 $\alpha$, stromal cell-derived factor $1 \alpha$.

Conflict of interest: The authors have declared that no conflict of interest exists. Citation for this article: J. Clin. Invest. 117:616-626 (2007). doi:10.1172/JCI28180. ligand interactions (16-18). The strength of the integrin-ligand interaction is regulated through changes in avidity and affinity of the integrins (19). Shear stress plays an important role in this process, as it counteracts the adhesion of the rolling cells $(20,21)$ and affects the density of adhesion factors $(22,23)$. In both LSS and OSS fields, these 2 factors enhance the uptake of inflammatory cells.

Recent studies indicate that avidity and affinity of integrins are modulated by chemokines $(17,24)$. Chemokines are a family of small, secreted proteins consisting of C, C-C, C-X3-C, and C-X-C subgroups based on the positioning of cysteine residues in their backbones (25). Data demonstrating a role for LSS or OSS in the regulation of chemokines come from a limited number of in vitro studies. These studies show a shear stress-dependent expression of monocyte chemoattractant protein-1 (MCP-1/CCL2) $(26,27)$ and growth-regulated oncogene $\alpha$ (GRO- $\alpha$ /CXCL8) (28). Chemokine specificity determines the type of inflammatory cells that migrate into the vessel wall. Hence, they might also modulate the phenotype of atherosclerotic lesions that develop in response to shear stress. The role of shear stress-regulated chemokine expression during atherogenesis still needs to be investigated. Therefore, the aim of the study is to characterize the chemokine expression pattern in shear stress-induced plaque formation.

\section{Results}

The effect of LSS and OSS on the development of atherosclerotic lesions. Cast placement around the common carotid artery reduced shear stress upstream and induced OSS downstream from the cast $(5,29)$. To study the effects of these shear stress fields on atherogenesis, a time series experiment was performed (time points 1, 3, and 9 weeks; $n=5$ mice per time point). No statistically significant differences in 
A

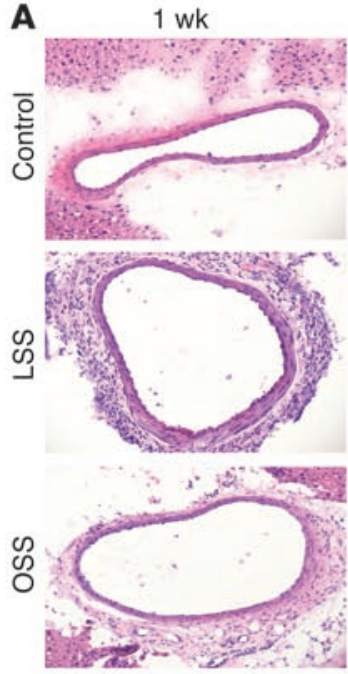

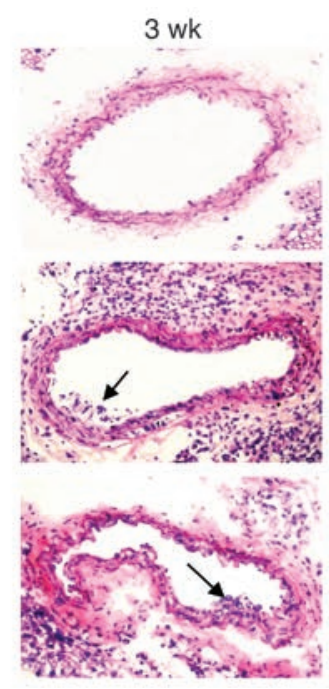

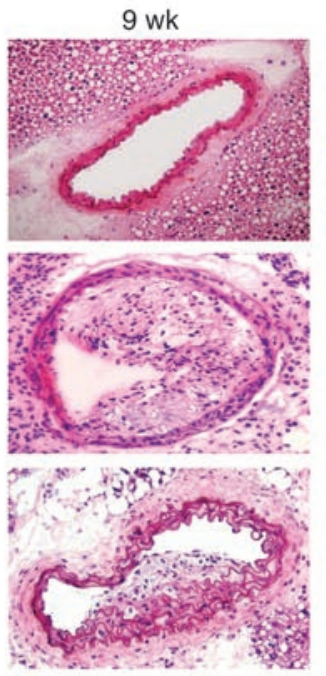

B

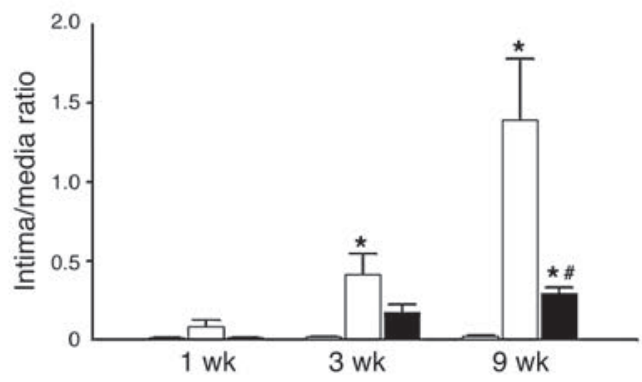

Figure 1

Representative H\&E-stained cross-sections of murine carotid arteries at different time points after cast placement in mice on a Western diet. (A) Sections from the OSS region, LSS region, and undisturbed region (control) 1, 3, and 9 weeks after cast placement. The arrows indicate accumulations of macrophages in fatty streaks (original magnification, $\times 200$ ). (B) Intima/media ratio in the control regions (gray bars), the LSS regions (white bars), and the OSS regions (black bars) at the 3 different time points. ${ }^{*} P<0.05$ versus control; ${ }^{*} P<0.05$ versus LSS.

the intimal area/media area ratio were observed between the different shear stress areas 1 week after cast placement due to minimal atherogenesis (Figure 1, A and B). At 3 weeks, the intimal/media ratio in the LSS region was significantly larger than that in the control region (Figure 1B), but the difference between the OSS and control regions was not statistically significant. Evaluation of the intimal/media ratio at 9 weeks showed lesions in the LSS area that were larger in size than those in the OSS (by 5-fold) and control (by 66 -fold) regions. The intimal/media ratio in the OSS region was also larger (by 14-fold) than in the control region at this time point. Furthermore, lesions that developed under LSS conditions contained larger necrotic cores underneath thinner fibrous caps than the lesions in the OSS regions. Therefore, the lesions in the OSS regions resembled the morphology of "stable", i.e., not ruptureprone, atherosclerotic lesions in humans (Supplemental Figure 1; supplemental material available online with this article; doi:10.1172/ JCI28180DS1). These findings are in accordance with previously obtained data (6).

Gene expression of specific chemokines characterizes LSS-induced plaque development. To investigate the effect of LSS and OSS on chemokine expression under proatherogenic conditions, we screened the linearly amplified pools of antisense
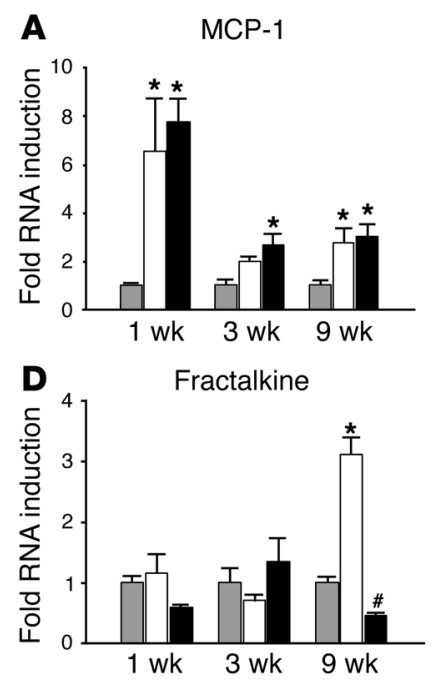

Figure 2
RNA from vessel segments exposed to the different shear stress conditions 1,3 , and 9 weeks after cast placement in $a p o E^{-/-}$mice. Because no plaques were present in the LSS or OSS regions after 1 week (Figure 1, A and B), the results obtained for this time point show the effect of shear stress alteration on the vessel wall without an existing atherosclerotic lesion. In addition, the 3-week time point allowed us to study the effect of shear stress on the carotid artery with an early atherosclerotic lesion. Finally, at 9 weeks after cast placement, the specific chemokine expression of advanced lesions was analyzed. We compared the response of the chemokines MCP-1, GRO- $\alpha / \mathrm{KC}$,
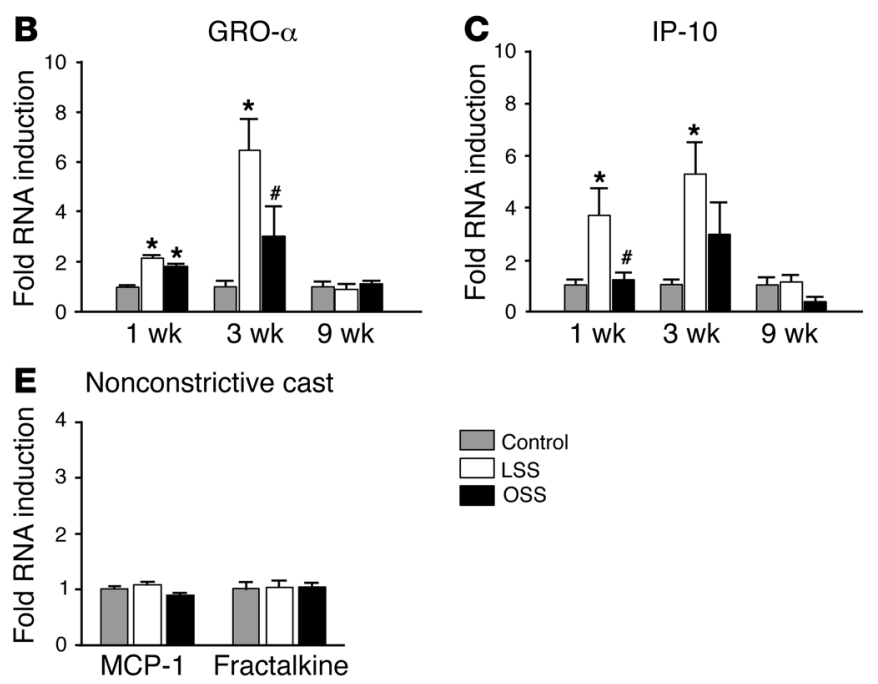

Validation of the expression levels of the upregulated chemokines. MCP-1 (A), GRO- $\alpha / \mathrm{KC}(\mathrm{B}), \mathrm{IP}-10$ (C), and fractalkine (D) expression levels were measured by QPCR using pooled nonamplified cDNA samples (5 animals per pool; $n=3$ ) at different time points (1,3, and 9 weeks) and different locations (LSS, control, and OSS regions). ${ }^{\star} P<0.05$ versus control; $\#<0.05$ versus LSS. (E) Placement of a nonconstrictive, control cast that does not alter the laminar shear stress did not induce changes in gene expression. 
A
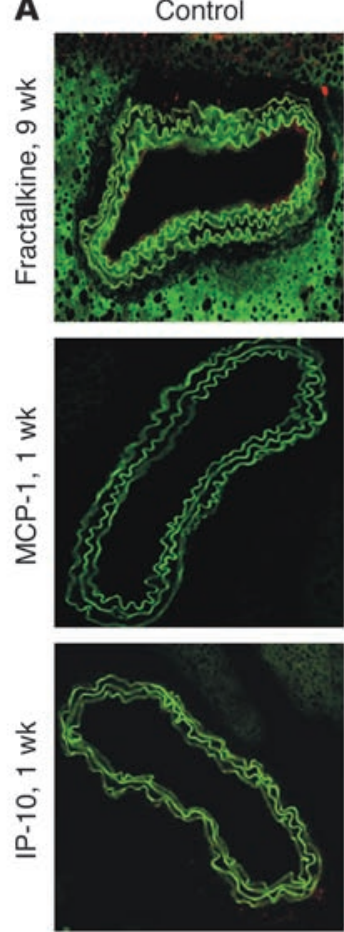

B

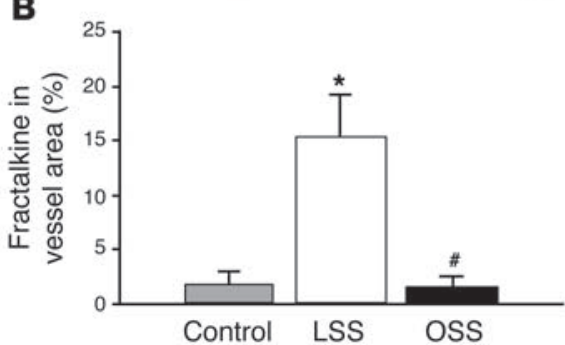

LSS
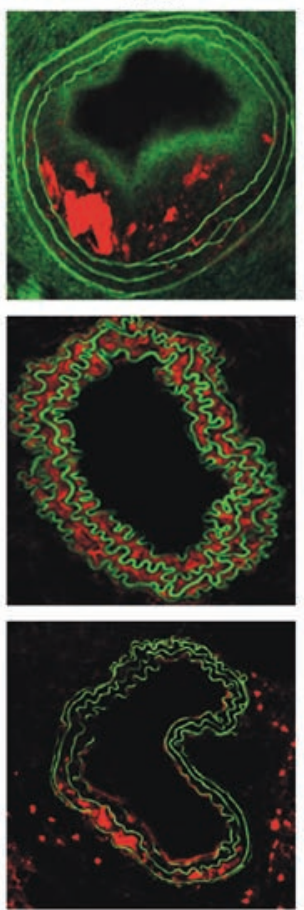

oss
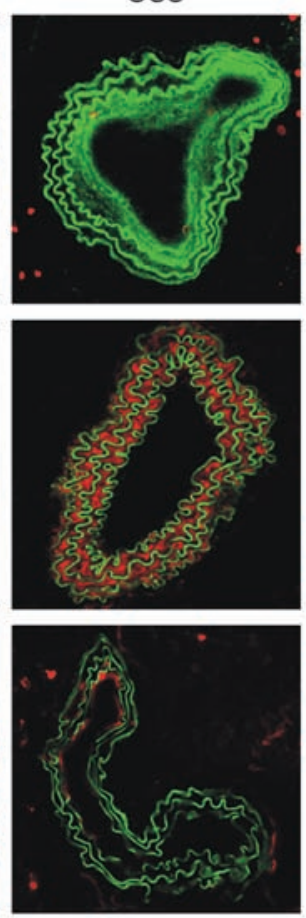

Detail, LSS area
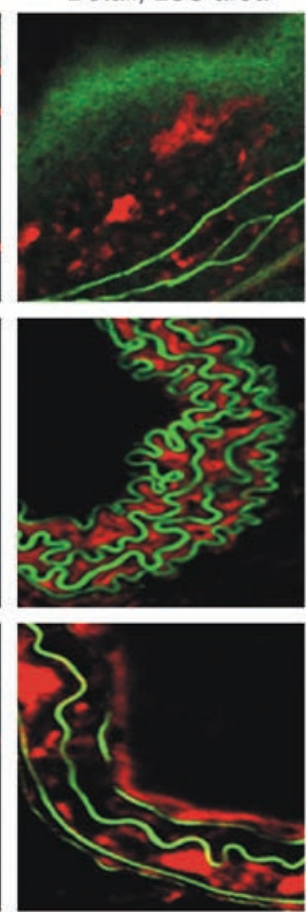
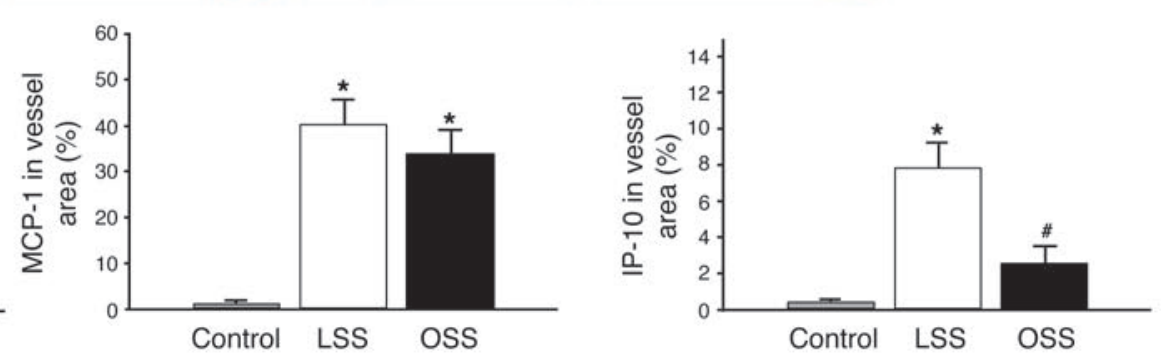

Figure 3

Confirmation of gene expression results of selected chemokines by immunohistological analysis of the protein levels in carotid arteries at different time points after cast placement in mice on a Western diet. (A) Representative cross-sections of murine carotid arteries stained for fractalkine 9 weeks after cast placement and MCP-1 and IP-10 1 week after cast placement, in the control, LSS, and OSS regions (original magnification, $\times 200)$. Chemokine expression is represented by a red signal. Green autofluorescence is from the elastic laminae and demarcates the intimal and medial areas. (B) Immunohistological quantification of the chemokine signals of the LSS, OSS, and control regions is shown. $n=5$. ${ }^{*} P<0.05$ versus control; ${ }^{\#} P<0.05$ versus LSS.

RANTES, eotaxin-1, EBI1 ligand chemokine (ELC), stromal cellderived factor $1 \alpha(\mathrm{SDF}-1 \alpha)$, neutrophil-activating protein-2 (NAP-2), IFN- $\gamma$-inducible protein-10 (IP-10), fractalkine, and C-C chemokine ligand 14 (CCL14) (all reported to be involved in atherosclerosis or to be regulated by shear stress; refs. $26,28,30$ ) on LSS and OSS stimuli at different time points. Of the 10 chemokines investigated in this study, 4 (MCP-1, GRO- $\alpha / \mathrm{KC}$, IP-10, and fractalkine) were upregulated in response to alterations in shear stress at different time points. No shear stress-responsive upregulation in the expression of the other chemokines (RANTES, eotaxin-1, ELC, NAP-2, SDF-1 $\alpha$, and CCL14) was observed (Supplemental Table 1). After 1 week, quantitative real-time PCR (QPCR) showed an increase in expression of the chemokines MCP- 1 and GRO- $\alpha / \mathrm{KC}$ in the altered shear stress compared with control regions. In addition, IP-10 was expressed at a higher level in the LSS region than in the OSS region (Supplemental Figure 2A). Three weeks after cast placement, the increased gene expression levels of MCP-1 were maintained in both the LSS and OSS regions, while GRO- $\alpha / \mathrm{KC}$ and IP-10 were only upregulated in the LSS region (Supplemental Figure 2B). Nine weeks after cast placement, the expression of MCP-1 and IP-10 returned to baseline levels (Supplemental Figure 2C). Of interest, fractalkine was only upregulated at this time point, and exclusively in the LSS regions.

Validation experiments. To verify the results obtained from the linearly amplified samples, we repeated the QPCR experiments with pooled samples of nonamplified RNA for those chemokines that were differentially upregulated. Essentially the same expression patterns were observed for MCP-1, GRO- $\alpha / \mathrm{KC}$, IP-10, and fractalkine (Figure 2, A-D) when we compared data from nonamplified RNA with data obtained with linearly amplified RNA. In particular, the expression patterns of fractalkine were very similar.

In order to exclude possible non-shear stress-related effects of the cast on chemokine expression, we performed control experiments with sham casts. These sham casts were made of the same material as the real cast but did not induce the alterations in shear 
A
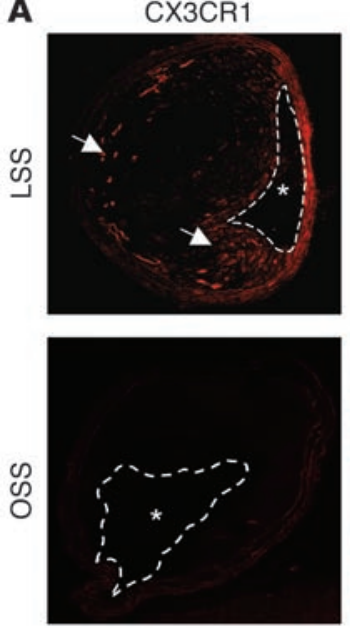

+ DAPI
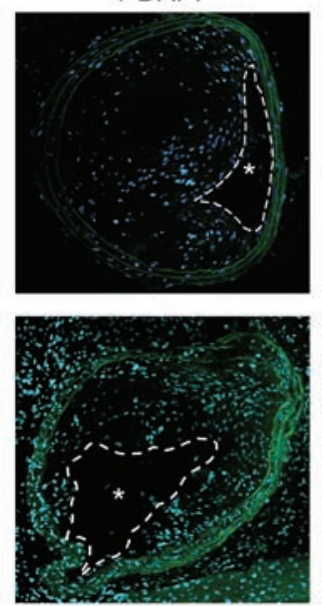

Detail
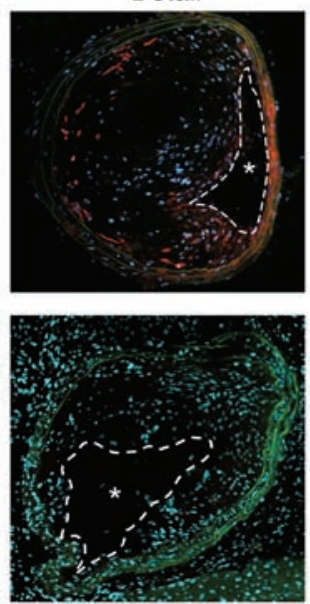

B

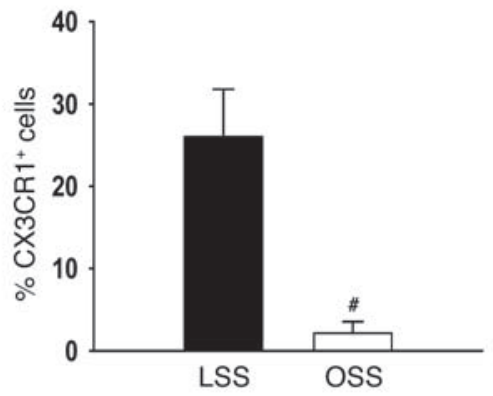

Figure 4

Accumulation of CX3CR1+ cells in the different shear stress-induced mature atherosclerotic lesions 9 weeks after cast placement. (A) Representative cross-sections of murine carotid arteries stained for CX3CR1 (red signal) and nuclei (blue signal). Arrows indicate CX3CR1+ cells. Asterisks indicate the position of the lumen, while the white dotted lines demarcate the boundaries between lumen and intima. (B) Immunohistological quantification of the chemokine/receptor signal in the LSS and the OSS region. $n=5$. $P<0.05$ versus LSS.

stress due to a nonconstrictive luminal geometry. Placement of the sham casts failed to cause lesion formation at locations either upstream or downstream from the device even after 9 weeks of instrumentation. Gene expression analysis on cDNA obtained from both locations did not reveal upregulation of MCP-1 or fractalkine (Figure 2E).

Total cholesterol levels in plasma were increased in animals on the atherogenic Western-type diet (1 week, $27.3 \pm 2.0 \mathrm{mmol} / 1 ; 3$ weeks, $22.4 \pm 0.9 \mathrm{mmol} / 1 ; 9$ weeks, $24.3 \pm 2.4 \mathrm{mmol} / \mathrm{l})$ compared with animals on chow $(10.9 \pm 3.0$ $\mathrm{mmol} / \mathrm{l})$. However, total cholesterol concentrations in animals treated with the cast or the nonconstrictive sham cast were not different ( 9 weeks cast, $24.3 \pm 2.4 \mathrm{mmol} / 1$ versus 9 weeks sham cast, $25.5 \pm 3.0 \mathrm{mmol} / \mathrm{l})$. Therefore, the observed variations in chemokine expression cannot be attributed to differences in plasma cholesterol levels.

Protein levels follow gene expression levels of selected chemokines. We evaluated the protein levels of a selected group of responsive chemokines. Fractalkine was studied because it was the only chemokine with elevated expression in the atherosclerotic lesions induced by LSS that showed the interesting plaque composition. Indeed, more intense fractalkine staining was found in the LSS region compared with the OSS and control regions (Figure 3, A and B) at 9 weeks, which is in accordance with the mRNA expression data. We also evaluated MCP-1, as the relevance of this chemokine for the pathology of atherosclerosis is well known (25). Immunohistological analysis of vessel segments at 1 week, which was the time point with the highest MCP-1 expression (see Figure 2), confirmed the upregulation of MCP-1 under both shear stress conditions (Figure 3, A and B). In addition, we selected one of the chemokines that were upregulated during early atherosclerosis development induced by LSS. The differences in protein level of IP-10 between the LSS and the OSS area 1 week after cast placement closely resemble the mRNA expression pattern (Figure 3, A and B).

Fractalkine receptor-expressing cells are present in the LSS-induced atherosclerotic plaques. From the above findings, we concluded that fractalkine is an interesting chemokine for further studies. We therefore assessed
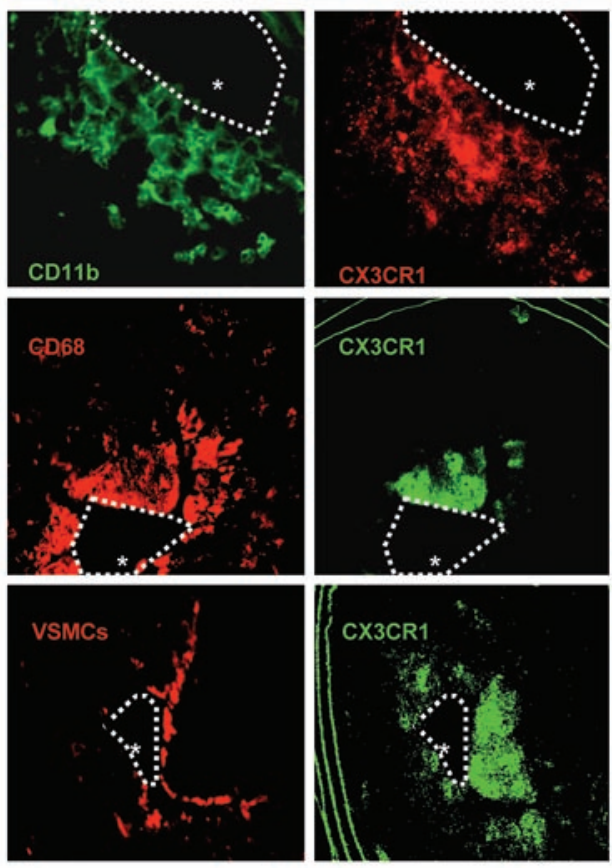

\section{Figure 5}

CX3CR1-expressing cells located in the advanced lesions are mainly monocytes and macrophages. Representative cross-sections of murine carotid arteries stained for CX3CR1 and CD11b (top row), CD68 (middle row), and VSMC $\alpha$-actin (bottom row). In the right column, overlays show the colocalization signal in yellow. Asterisks indicate the position of the lumen, while the white dotted lines demarcate the boundaries between lumen and intima. Double staining for the identification of CX3CR1-expressing cell types was repeated in 4 different experiments. 
LSS -

A

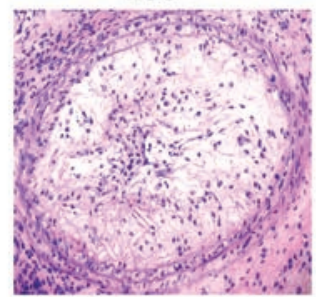

B

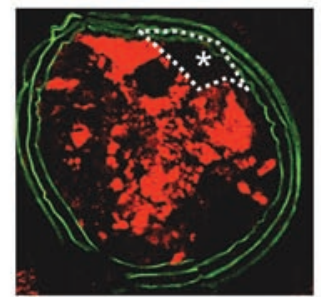

C

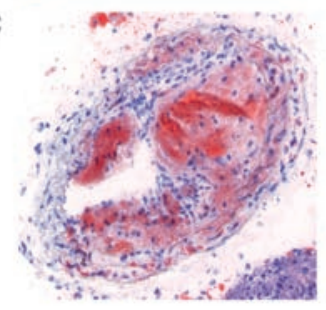

D

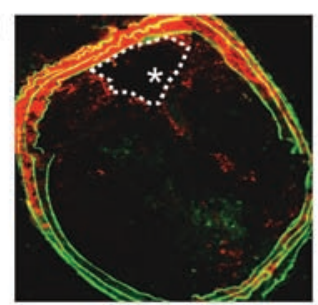

E

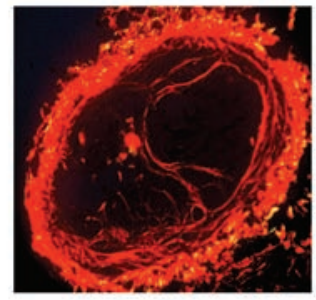

F

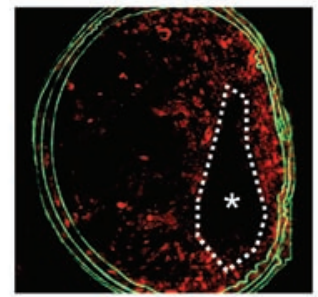

LSS +
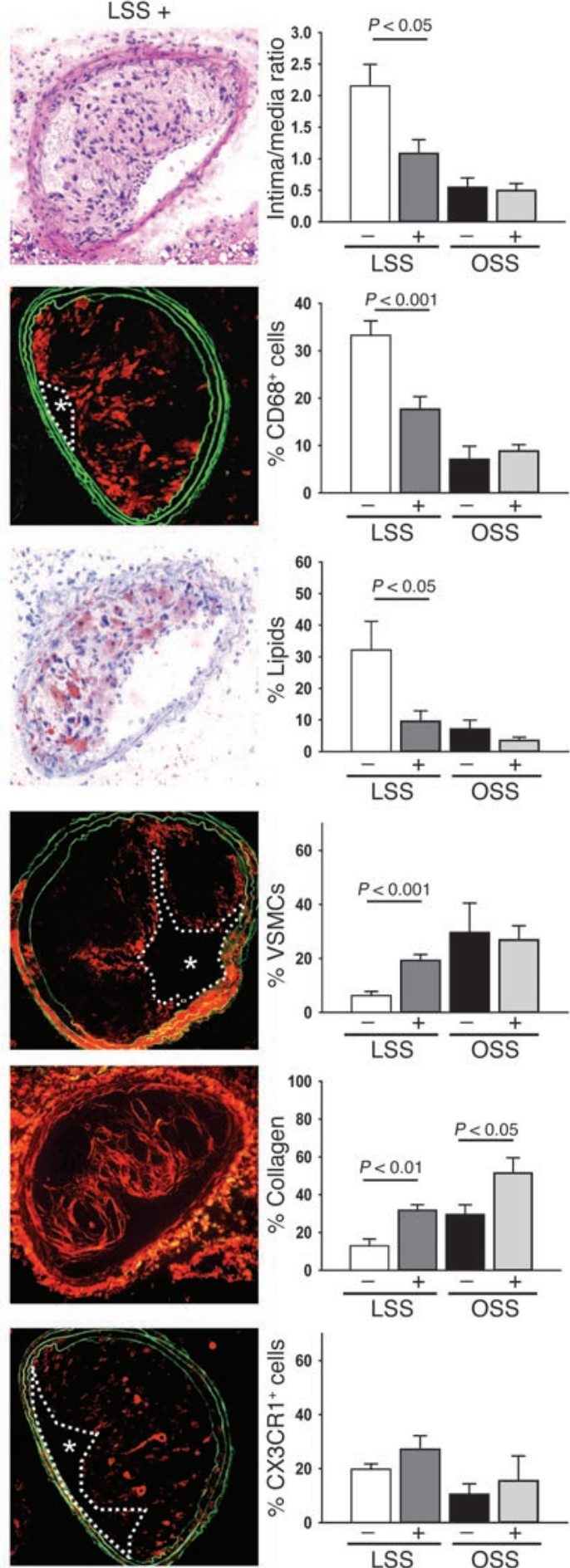
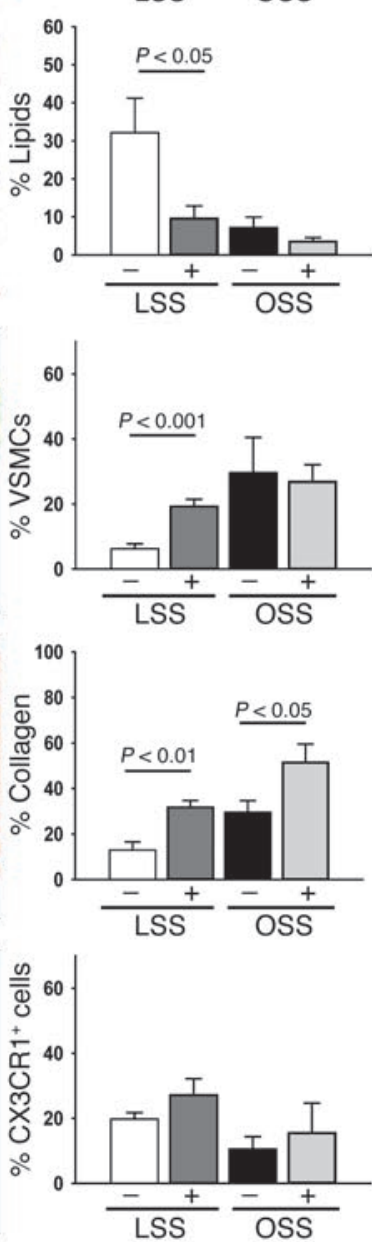

\section{Figure 6}

Fractalkine function was inhibited during cast-induced atherogenesis in $a p o E^{-/-}$mice by administration of a neutralizing antibody from week 6 until week 9 . Representative cross-sections are shown stained for: lesion morphology by H\&E (A), macrophages (B), lipids (C), VSMCs (D), collagen (E), and fractalkine receptor (CX3CR1) (F). Asterisks indicate the position of the lumen, while the white dotted lines demarcate the boundaries between lumen and intima. Bar diagrams represent the lesion area and quantification of the (immuno)histological analysis of the different plaque components. The percentages of positive area at 9 weeks in the intima in the LSS and the OSS regions are indicated, comparing the control antibody group $(-)$ with the anti-fractalkine antibody group $(+) . n=8$. ${ }^{\star} P<0.05$ versus control antibody group. the effect of LSS and OSS on the accumulation of cells expressing C-X3-C chemokine receptor 1 (CX3CR1; i.e., the fractalkine receptor) in advanced lesions in order to determine its contribution to the pathology of the LSS lesions. CX3CR1-expressing cells were detected in the intimal area of lesions situated in the LSS region, while in the lesions in the OSS regions, CX3CR1-expressing cells were detected at extremely low levels (Figure 4, A and B). Further analysis of the atherosclerotic lesions located in the LSS area showed that many cells positive for CX3CR1 were also positive for the monocyte/macrophage markers CD11b and CD68 in the lesions (Figure 5, upper and middle rows, respectively). Double staining for CX3CR1 and VSMC $\alpha$-actin did not show any obvious evidence of smooth muscle cells expressing the fractalkine receptor (Figure 5, bottom row). Serial cross-sections showed no general overlap of these CX3CR1 ${ }^{+}$cells with the fractalkine $^{+}$areas, indicating that most of these receptor-positive cells do not produce the fractalkine ligand (Supplemental Figure 4). 

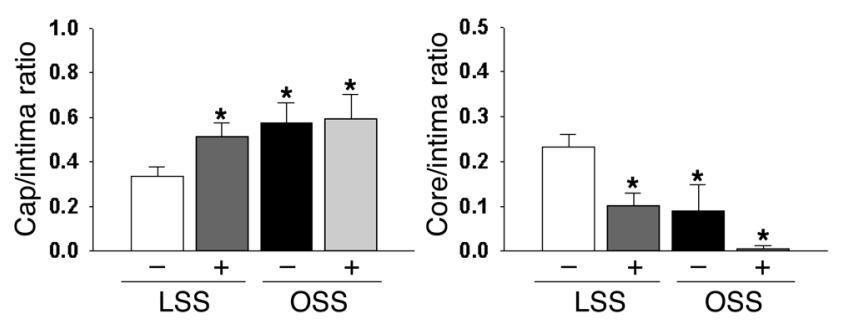

Inbibition of fractalkine function restrains growth and stabilizes atherosclerotic plaques induced by LSS. To evaluate the functional implications of the findings described above, we studied the possible effect of fractalkine inhibition on systemic effects, plaque composition, and plaque vulnerability. Microscopic inspections of H\&E-stained sections of liver, lung, spleen, and draining lymph nodes of the carotid artery did not reveal any abnormalities in animals treated with the rabbit polyclonal IgG neutralizing antibody against fractalkine in the fractalkine group or the rabbit polyclonal IgG control antibody in the control group (data not shown). Furthermore, FACS analysis on spleen-derived and peripheral blood leukocytes showed no differences in the numbers of $\mathrm{CD}^{+}$cells or in the $\mathrm{CD}^{+} / \mathrm{CD}^{+}$cell ratios between the 2 groups (data not shown).

Animals in the fractalkine inhibition group showed a $50 \%$ reduction in plaque area in LSS regions only (Figure 6A), i.e., in the regions with a differential upregulation of fractalkine. In addition, fractalkine inhibition changed plaque composition - with the exception of collagen content - exclusively in the LSS-induced plaques: treatment with fractalkineneutralizing antibodies approximately halved $\mathrm{CD} 68^{+}$macrophage content and lipid accumulation in this type of atherosclerotic plaque compared with the control group and doubled the number of VSMCs (Figure 6, A-D). However, in the OSS regions, no differences between the fractalkine and the control group were observed in size and content of the plaques. In both the LSS and OSS regions, treatment with anti-fractalkine antibodies resulted in increased collagen content (Figure 6E). In both shear stress regions, no effect was found on the number of CX3CR $1^{+}$cells in response to fractalkine antibody treatment (Figure 6F).

Morphometric evaluation of the plaques in the LSS region and the OSS region show that the relative cap area

\section{Figure 8}

\section{Figure 7}

The effect of fractalkine inhibition on cap thickness and the size of the necrotic core in the LSS and OSS lesions. Quantification of cap thickness (left) and the necrotic core (right) relative to total intimal area of the LSS and the OSS regions, comparing the control antibody group with the anti-fractalkine antibody group. $n=8$. ${ }^{*} P<0.05$ versus LSS without antibody treatment.

(cap/intima ratio) and the relative necrotic core size (core/intima ratio) were both approximately 2 -fold smaller in the atherosclerotic lesions induced by LSS than by OSS (Figure 7). Treatment with anti-fractalkine antibodies resulted in an increase in cap thickness and decreased the size of the necrotic core in the LSSinduced lesions (Figure 7).

Fractalkine might also affect the infiltration of other types of immune component cells involved in atherogenesis. Therefore,

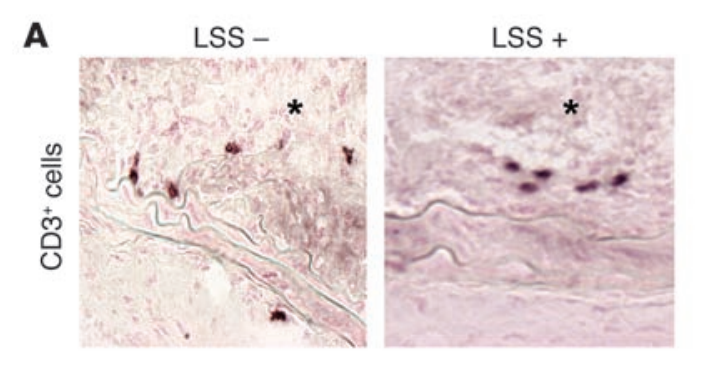

B

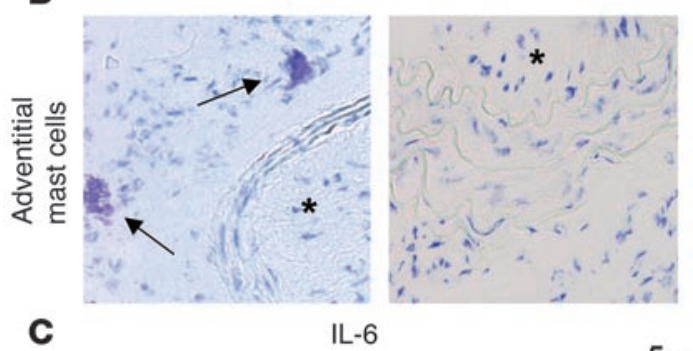

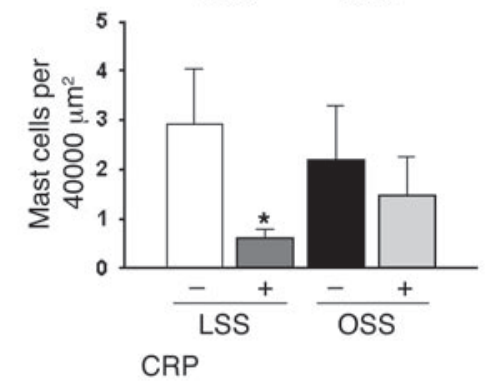
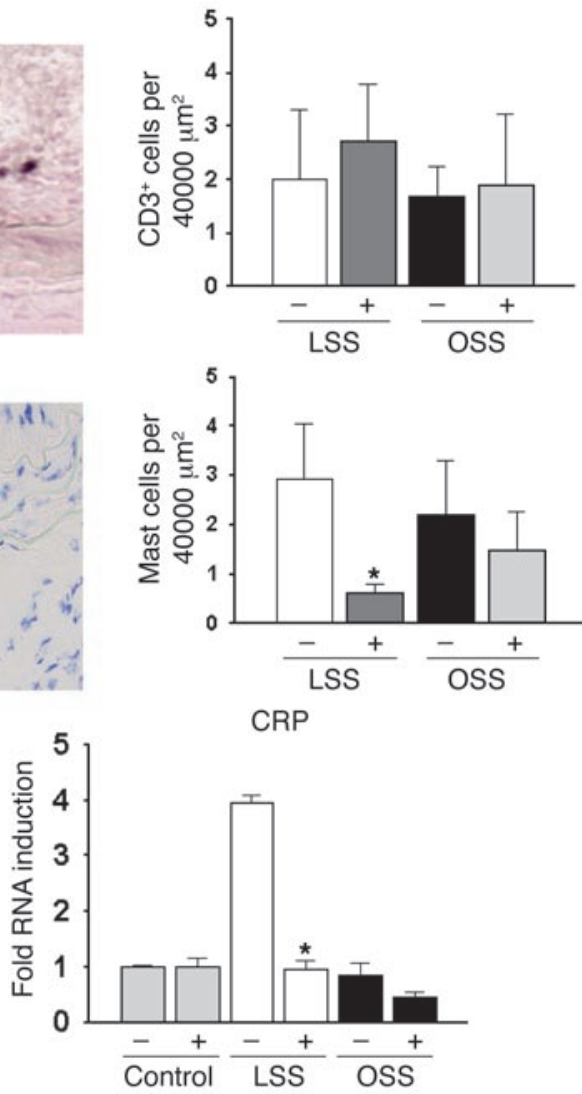

The effect of fractalkine inhibition on T cells (A) and mast cells infiltration (B) and IL-6 and C-reactive protein (CRP) expression (C) in the LSS and OSS lesions. Left panels: representative cross-sections taken from the LSS lesions from apo $E^{-/-}$mice 9 weeks after cast placement with administration of rabbit IgG control antibodies or with the neutralizing antibody against fractalkine. Cross-sections were stained for $\mathrm{CD}^{+} \mathrm{T}$ cells $(\mathbf{A})$ and mast cells using toluidine blue $(\mathbf{B})$ (original magnification, $\times 200)$. CD3 ${ }^{+} \mathrm{T}$ cells and mast cells are indicated by arrows. Asterisks indicate the intimal area. Right panels: The number of positive cells counted in the intima (CD3+ $\mathrm{T}$ cells) and adventitia (mast cells) in the LSS and OSS lesions, with administration of rabbit IgG control antibodies or with the neutralizing antibody against fractalkine. $n=6$. ${ }^{\star} P<0.05$ versus control antibody group. (C) Expression levels of CRP and IL-6 in carotid arteries at 9 weeks after cast placement in mice on a Western diet in response to fractalkine inhibition. Expression profiles were measured by QPCR using amplified RNA samples (10 animals per pool; $n=3$ ). In the control antibody group, IL-6 and CRP levels in the LSS region were significantly upregulated versus the control and OSS regions. ${ }^{*} P<0.05$ versus anti-fractalkine antibody group. 

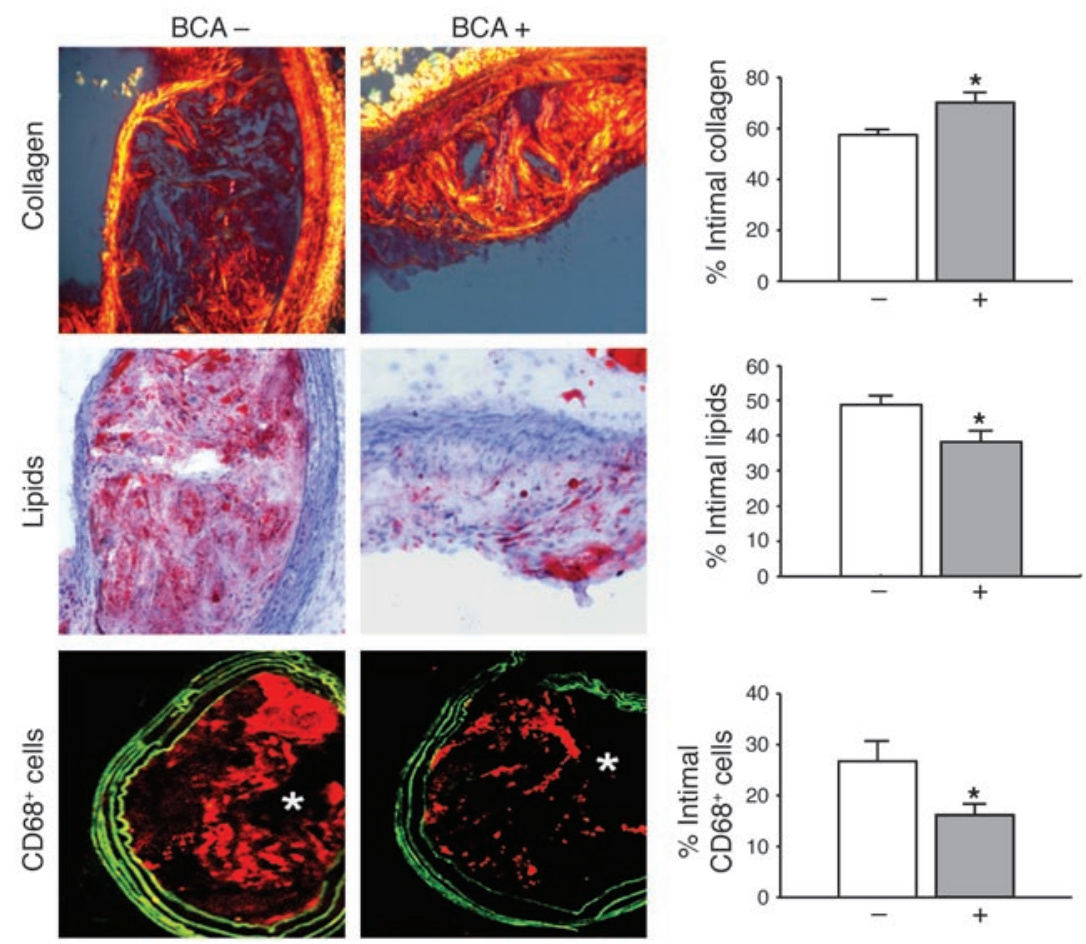

\section{Figure 9}

Fractalkine function was inhibited during atherosclerosis development in the BCAs in apoE $E^{-/-}$ mice on a Western diet by administration of a neutralizing antibody from week 6 until week 9 . Representative cross-sections are shown stained for: collagen (upper panels), lipids (middle panels), and macrophages (lower panels). Asterisks indicate the position of the atherosclerotic lesions. Bar diagrams represent quantification of the (immuno-)histological analysis of the different plaque components. The percentages of positive area at time point 9 weeks in the intima of the $\mathrm{BCAs}$ are indicated, comparing the control antibody group with the fractalkine antibody group. $n=6 .{ }^{*} P<0.05$ versus control antibody group. we analyzed the lesions induced by LSS or OSS from mice treated with the anti-fractalkine antibodies or with control antibodies for the accumulation of $\mathrm{CD}^{+}$(pan-T cell marker) T cells, mast cells, and NK cells. We found that fractalkine inhibition had no effect on $\mathrm{CD}^{+} \mathrm{T}$ cell accumulation (Figure 8A). However, inhibition of fractalkine function strongly reduced the numbers of mast cells in the adventitia of the plaques induced by LSS (Figure 8B). For the detection of NK cells, we used the anti-mouse Ly49G2 antibody 4D11, which has no cross-reaction with macrophages and has been used previously for the detection of NK cells in murine plaque areas (31). In the atherosclerotic lesions induced by either LSS or OSS, NK cells were scarce, limited to 1-2 cells per lesion, and fractalkine inhibition did not elicit any effects (Supplemental Figure 3).

Gene expression analysis of the different vessel segments at the 9-week time point revealed increased expression of the proinflammatory mediators C-reactive protein and IL-6 in the LSS versus the OSS regions in the control group. Fractalkine inhibition reduced gene expression of CRP and IL-6, as expression of these genes in the LSS areas was significantly decreased in response to antibody treatment (Figure 8C).

Subsequently, we investigated whether the effect of fractalkine inhibition is restricted to the lesions induced by the cast model. Therefore, the composition of the atherosclerotic lesions in the brachiocephalic arteries (BCAs) was analyzed. We indeed found effects of fractalkine inhibition on BCA lesions. Treatment with neutralizing antibodies against fractalkine reduced lipid and macrophage accumulation in the intimal area. It also increased the collagen content in these BCA lesions (Figure 9). No significant differences in the percentage of intimal VSMCs and lesion size were found, but a trend toward a reduction in intimal area in response to the treatment (control group, $0.11 \pm 0.01 \mathrm{~mm}^{2}$ versus fractalkine inhibition group, $0.08 \pm 0.01 \mathrm{~mm}^{2}$ ) was observed.

\section{Discussion}

The main findings of this study are that (a) the development of atherosclerotic plaques induced by LSS is characterized by a specific expression of GRO- $\alpha / \mathrm{KC}$, IP-10, and fractalkine; and (b) fractalkine determines the growth and the composition of this type of atherosclerotic lesion.

The high mortality associated with atherosclerosis can be ascribed to a large extent to rupture of vulnerable plaques $(32,33)$. The morphology of vulnerable plaques consists of a characteristic triad: a large lipid core, a thin fibrous cap, and an accumulation of inflammatory leukocytes. This inflammatory component is prominent in plaques with a vulnerable phenotype, and it has been postulated that activation of the immune system is an important condition for the development of vulnerable plaques (7-11). Accumulation of inflammatory cells in plaques has been examined extensively, and a cascade model has emerged from these studies. This model describes rolling, arrest, and migration as a sequential process (9). Arrest of inflammatory cells on the endothelial layer in an arterial flow field, as occurred in our experiments, is due to an increased clustering on the cell surface (avidity) and increased affinity of integrins for their ligands $(20,21)$. Both processes are strongly modulated by chemokines $(17,24)$. However, the role of shear stress in the expression of chemokines is largely unknown.

We studied the effect of shear stress on chemokine expression, and thus potential leukocyte recruitment during plaque formation, performing a screening of 10 chemokines that are known to be involved in the pathology of atherosclerosis or are known to be responsive to shear stress. Interestingly, 4 of the selected genes, MCP-1, GRO- $\alpha / \mathrm{KC}$, IP-10, and fractalkine, were upregulated. These were studied in more detail.

MCP-1 accumulation has been demonstrated in human atherosclerotic lesions and in plaques induced in animal models $(34,35)$. It is involved in monocyte/macrophage recruitment, and knockout 
studies indicate an important role in atherogenesis (25). In the present study, MCP-1 expression levels did not differ whether lesions were induced by LSS or OSS. These findings are in accordance with in vitro studies showing a role for both LSS and OSS in MCP-1 production by endothelial cells $(26,27)$. This lack of differential expression and the decline in MCP-1 expression after the first week of shear stress stimulation may imply that the interaction of MCP-1 with its receptor, CCR2, is important predominantly during early atherogenesis and may not play an important role in determining the phenotype of the advanced atherosclerotic lesion. Recent studies with CCR2-deficient mice indicate that early reconstitution of CCR2 ${ }^{+}$cells prevents the effects of CCR 2 deficiency, while this effect is absent during later phases (36), similar to the present findings.

Two other genes (IP-10 and GRO- $\alpha / \mathrm{KC}$ ) were upregulated in the early phase of LSS-induced plaque formation. IP-10 is a chemoattractant for monocytes (37) and T lymphocytes (38) and is induced by production of IFN- $\gamma$ (39). Only a small number of studies have identified this chemokine in relation to vascular disease. However, as IFN- $\gamma$ has been associated with vulnerable plaque formation (40), this chemokine may be of importance. Indeed, increased serum levels of IP-10 have been associated with coronary artery disease in patients (41).

GRO- $\alpha / \mathrm{KC}$ and its receptor CXCR-2 are involved in the accumulation of macrophages during atherogenesis, and high-level expression of CXCR-2 by macrophages is detected in advanced lesions of patients and LDL receptor-deficient mice (42). Surprisingly, increased GRO- $\alpha / \mathrm{KC}$ expression was only detected at earlier time points ( 1 and 3 weeks). This implies that GRO- $\alpha / K C-C X C R-2$ interaction is mainly involved during the early events of LSSinduced plaque formation.

Of the upregulated chemokines, fractalkine is the only one that is exclusively expressed in the LSS-induced atherosclerotic plaque. Fractalkine is a unique chemokine that acts not only as a chemoattractant but also as an adhesion molecule expressed on endothelial cells upon stimulation (43). The fractalkine receptor CX3CR1 is expressed on monocytes, macrophages, lymphocytes, VSMCs (44), and endothelial cells, where its expression is regulated by IFN- $\gamma(45,46)$. Functional studies in mice indicate that CX3CR1 or fractalkine deficiency results in smaller atherosclerotic lesions, associated with a reduction in the number of macrophages and foam cells (47-49). In the present study, we observed more $\mathrm{CX} 3 \mathrm{CR} 1^{+}$cells in the plaques with the distinct composition located in the LSS region as compared with the plaques found in the OSS regions, suggesting that there is an effect of fractalkine expression on cell recruitment. Subsequent qualitative analysis of CX3CR $1^{+}$ cells identified predominantly monocytes and macrophages and few VSMCs colocalizing with the fractalkine receptor. It is postulated that monocytes may enter the atherosclerotic lesion from the adventitial side. However, in the lesions induced by LSS, cells positive for CD11b, which is a cell surface marker for monocytes that is downregulated during their differentiation into macrophages (50), were mainly detected just underneath the endothelium (Figure 5). Additional studies in which we stained the lesions for the myeloid-committed progenitor cell marker er-mp53 (51) showed er-mp53 $3^{+}$cells mainly located in close proximity to the lumen (data not shown). We conclude from these data that plaque macrophages enter the lesions that are induced by LSS via the luminal side.

Inhibition of fractalkine-CX3CR1 interaction decreased plaque growth and altered the composition of the atherosclerotic plaques within the LSS regions. The exact mechanism is presently unknown but might involve a reduced accumulation of macrophages and foam cells, derived from CX3CR $1^{\text {high }}$ monocytes. In addition, the lesions contained increased amounts of extracellular matrix (collagen) and VSMCs, thereby increasing collagen synthesis, when compared with the lesions of the control group. Importantly, inhibition of fractalkine-CX3CR1 interaction had only minor effects on the lesions induced by OSS. This might indicate a role for LSS in the fractalkine-mediated effects. However, as fractalkine upregulation was only observed in the LSS plaques 9 weeks after cast placement, other factors may be involved. For instance, extreme narrowing of the luminal area during later phases of plaque growth might have increased shear stress locally, and this increase in shear stress might have provided the trigger for fractalkine expression. In addition, inflammatory mediators (e.g., IFN- $\gamma$ ) released from within the vessel wall during atherogenesis induced by LSS might also be responsible for the upregulation of fractalkine. Nevertheless, expression of fractalkine remains a distinct (indirect) effect of LSS induction of atherosclerosis, and the results of the functional experiments presented in this study are important, as they clearly show the critical role of fractalkine in determining the composition of advanced atherosclerotic lesions.

Recent in vitro findings obtained from flow chamber assays indicate that fractalkine contributes to platelet activation and adhesion and could therefore play an important role in increasing thrombogenesis in atherosclerosis (52). We studied platelet aggregation in the different shear stress lesions in response to fractalkine inhibition by immunohistochemistry, staining for P-selectin. Platelet aggregations were detected in both the LSSand OSS-induced lesions. However, these vascular pathologies were also observed in fractalkine antibody-treated animals. The platelets were activated, as platelet-leukocyte conjugates were formed (Supplemental Figure 5, A and C) lining the endothelium. Occasionally, large, platelet-rich thrombi were detected in the lumen (Supplemental Figure 5B). As activated endothelial cells also produce P-selectin, we double-stained with antibodies directed against von Willebrand factor (green signal). The results show that these defined histological structures positive for P-selectin were indeed platelet aggregations lying on top of von Willebrand factor-positive endothelial cells (Supplemental Figure 5D). These data indicate that fractalkine might not play a significant role in (micro)thrombogenesis in vivo in the context of atherosclerosis. Besides, increased thrombogenesis does not seem to be a prominent mechanism to explain the fractalkine effects.

The finding that fractalkine inhibition results in a decreased accumulation of mast cells in the adventitia of the LSS-induced lesions is interesting, as it has been shown by Virmani and coworkers that mast cells infiltrate the adventitia of atherosclerotic vessel segments during plaque development in humans (53). Accumulation of mast cells in the adventitia was also increased in the coronary arteries of patients who died of acute myocardial infarction (54). It was speculated that the release of histamine and leukotrienes by activated mast cells in the adventitia could cause constriction of the atherosclerotic vessels, leading to acute coronary syndrome (55). Fractalkine has been shown to be involved in the recruitment of mast cells in skin tissue (56) and in the airway of patients with asthma (57). Our study now shows that chemotaxis of mast cells into the adventitia of atherosclerotic vessel segments is (partly) regulated by fractalkine. The inhibition of fractalkine function could therefore potentially provide an additional therapeutical advantage. 
In the studies conducted on the atherosclerotic lesions of the BCA, we showed that the effect of fractalkine blockage is not restricted to lesions induced by the cast and that this chemokine is also involved in the progression of the disease at naturally occurring predilection sites. The effects of fractalkine are mostly on atherosclerotic plaques with a distinct composition supporting a less "stable" phenotype, as both the LSS-induced lesions (6) and the BCA lesions (58) show the occurrence of spontaneous intraplaque hemorrhages, which are rare at other atherosclerosis-prone locations of the arterial tree, whereas the effects of fractalkine inhibition on the lesions induced by OSS were limited.

A limitation of the current study is that by using whole homogenates from vessel tissue, we cannot distinguish in the mRNA data between changes in activation/expression level per individual cell and changes in the number of cells expressing the chemokine of interest. There is a clear relation and effect of these identified chemokines on plaque composition. It would be interesting to assess whether increased chemokine expression is derived from an activated subpopulation of intimal cells. Currently, we are conducting a large study in which we isolate specific cell types and subpopulations in the LSS and OSS lesions to evaluate their state of activation. In this study, we used a model for shear stress alteration, developed in-house, to induce 2 types of atherosclerotic lesions. Although the lesions triggered by LSS show a less "stable" phenotype than the lesions induced by OSS, no plaque rupture was detected. Until now, actual plaque rupture has not been demonstrated unambiguously in any animal model. We did observe, however, that the atherosclerotic lesions in the LSS area in our model were more susceptible to intraplaque hemorrhages. In addition, morphometric analysis of the LSS and the OSS regions indicated that they differ in fibrous cap thickness and necrotic core size. Taken together with the distinct difference in plaque composition between the atherosclerotic lesions induced by the 2 types of shear stress, these results allow us to conclude that the cast model is suitable for investigating the role of chemokines in determining plaque phenotype.

In conclusion, the present study indicates that different shear stress fields have yielded different plaque compositions associated with different responses in chemokine expression. LSS induces the development of larger lesions of which the composition resembles lesions in humans with a less "stable" phenotype. These lesions are characterized by increased expression of multiple chemokines (GRO- $\alpha / \mathrm{KC}$, fractalkine, and IP-10) compared with the OSSinduced lesions. Fractalkine appears to be crucial in the development of the distinct plaque phenotype that is induced by LSS. Therefore, stabilization of this type of atherosclerotic plaque in patients by inhibiting fractalkine-fractalkine receptor interaction might prove to be an effective therapy.

\section{Methods}

Mice. Mice deficient in apoE (apoE $E^{-/-}$mice) in a C57BL/6J background were obtained from The Jackson Laboratory. Animals 15-20 weeks of age were assigned randomly to 1 of the 3 time points (see below). During the experimental period, all animals were fed a Western-type diet consisting of $15 \%$ (wt/wt) cocoa butter and $0.25 \%$ (wt/wt) cholesterol (Diet W; Hope Farms). After a 2-week period of Western diet, shear stress in the right common carotid artery was altered by cast placement, as previously described (5). In short, the animals were anesthetized with isoflurane, and the anterior cervical triangles were accessed by a sagittal anterior neck incision. Both halves of the cast were placed around the right common carotid artery and fixed with a suture; wounds were closed; and the animals were allowed to recover. Animals with cast implants were sacrificed at 1,3 , and 9 weeks after surgery.

To study the effects of fractalkine blockage on plaque development, we injected $a p o E^{-1-}$ animals in the tail artery 3 times a week with $25 \mu \mathrm{g}$ of neutralizing rabbit anti-mouse fractalkine antibody (eBioscience) or rabbit IgG isotype control antibody (eBioscience). Mice received the injections from 6 to 9 weeks after cast placement, when the animals were sacrificed. All animal experiments were performed according to institutional and national regulations and approved by the Institutional Review Board on Animal Experiments of the Erasmus University Medical Center.

Gene expression analysis. Freshly isolated vessel segments were each divided into 3 different regions $(0.5 \mathrm{~mm}$ proximal from the cast, $1 \mathrm{~mm}$ in the cast, and $0.5 \mathrm{~mm}$ distal to the cast) and pooled per region $(n=10)$. Total RNA from the samples was extracted using the RNeasy kit (QIAGEN) and was linearly amplified following an Eberwine T7 protocol (59) or directly reversed transcribed into $\mathrm{cDNA}$ as indicated. QPCR reactions were performed using a real-time fluorescence determination in the iCycler iQ Detection System (Bio-Rad). Primers were designed for MCP-1, GRO- $\alpha$, RANTES/CCL5, eotaxin-1/CCL11, ELC/CCL19, SDF-1 $\alpha$ /CXCL12, NAP-2/ CXCL7, IP-10/CXCL10, fractalkine/CX3CL1, and CCL14. Target gene mRNA levels were expressed relative to the housekeeping gene hypoxanthine guanine phosphoribosyl transferase (HPRT) as an endogenous control (for primer sequences; see Supplemental Table 2).

Cholesterol analysis. Blood samples were taken under anesthesia by orbital puncture at the time of sacrifice. Total plasma cholesterol concentrations were measured enzymatically using a commercially available kit (kit 236691; Roche Diagnostics).

Histology. Six-micrometer cryosections of the right and the left common carotid arteries or BCAs were obtained as described previously (6). Sections equally spaced throughout the arteries at $120-\mu \mathrm{m}$ intervals were used for analyses. Macrophages were detected using anti-CD68 antibodies (Santa Cruz Biotechnology Inc.) and smooth muscle cells by antibodies against VSMC $\alpha$-actin (Sigma-Aldrich). Lipid deposition was analyzed with oil red O staining and collagen with picrosirius red. Fractalkine, MCP-1, IP-10 (Sigma-Aldrich), and CX3CR1 polyclonal antibodies (Santa Cruz Biotechnology Inc.) and R594- or FITC-labeled secondary antibodies (Invitrogen) were used for analysis with a Zeiss LSM 510 LNO inverted laser scanning confocal microscope.

Statistics. Data analysis was performed using a microscopy image analysis system (Impak C, Clemex Vision Image Analysis System). Intimal/media ratio was analyzed on H\&E-stained sections. In order to quantify the expression of different chemokines in the vessel, the area positive for the specific staining was expressed as a percentage of the total area enclosed by the external elastic lamina and the lumen. To assess the percentage of the different plaque components, the area within the intima positive for each stain was expressed as a percentage of the total intimal area. Results from the crosssections from one specific region of shear stress, i.e., LSS, OSS, and control regions, were averaged. For the morphometric analysis, the fibrocellular cap region was determined from collagen staining by picrosirius red and was expressed as a percentage of the total intimal area (cap/intima ratio) (60). The necrotic core region was determined as an acellular area of the lesion (core/intima ratio) (60). Area was determined from H\&E-stained sections and calculated as the total acellular area lacking in nuclei and cytoplasm. The necrotic core area was differentiated from regions of dense fibrous scars according to the presence of macrophage debris. Statistical analysis was performed using 1 ANOVA followed by Tukey's procedure. Data are presented as mean \pm SEM. $P$ values less than 0.05 were considered significant.

\section{Acknowledgments}

We would like to thank Fadime Doran and Meryem Algür for excellent technical assistance. This work was supported by grants from 
the Translational Research Program of the Erasmus University Medical Center and by the foundation Lijf en Leven, The Netherlands. Rob Krams is a recipient of an established investigatorship of the Netherlands Heart Foundation (grant 2002T045). Hetty C. de Boer was supported the Netherlands Heart Foundation (grant NHS 2005B106). Caroline Cheng was financially supported by the Interuniversity Cardiology Institute of the Netherlands (ICIN-33).
Received for publication February 9, 2006, and accepted in revised form December 19, 2006.

Address correspondence to: Rob Krams, Erasmus MC, Room Ee2216, Dr. Molewaterplein 40, 3015 GD Rotterdam, The Netherlands. Phone: 31-10-4087308; Fax: 31-10-4089494; E-mail: r.krams@erasmusmc.nl.
1. Cheng, C. 2006. Atherosclerotic lesion size and vulnerability are determined by patterns of fluid shear stress. Circulation. 113:2744-2753.

2. Williams, H., Johnson, J.L., Carson, K.G., and Jackson, C.L. 2002. Characteristics of intact and ruptured atherosclerotic plaques in brachiocephalic arteries of apolipoprotein E knockout mice. Arterioscler. Thromb. Vasc. Biol. 22:788-792.

3. Pedersen, E.M., et al. 1999. Distribution of early atherosclerotic lesions in the human abdominal aorta correlates with wall shear stresses measured in vivo. Eur. J. Vasc. Endovasc. Surg. 18:328-333.

4. Wentzel, J.J., et al. 2001. Shear-stress and wall-stress regulation of vascular remodeling after balloon angioplasty: effect of matrix metalloproteinase inhibition. Circulation. 104:91-96.

5. Buchanan, J.R., Jr., Kleinstreuer, C., Truskey, G.A., and Lei, M. 1999. Relation between non-uniform hemodynamics and sites of altered permeability and lesion growth at the rabbit aorto-celiac junction. Atherosclerosis. 143:27-40.

6. Stone, P.H., et al. 2003. Effect of endothelial shear stress on the progression of coronary artery disease, vascular remodeling, and in-stent restenosis in humans: in vivo 6-month follow-up study. Circulation. 108:438-444.

7. Cheng, C., et al. 2005. Shear stress affects the intracellular distribution of eNOS: direct demonstration by a novel in vivo technique. Blood. 106:3691-3698.

8. de Nooijer, R., et al. 2004. Overexpression of IL-18 decreases intimal collagen content and promotes a vulnerable plaque phenotype in apolipoprotein-Edeficient mice. Arterioscler. Thromb. Vasc. Biol. 24:2313-2319.

9. Dickson, B.C., and Gotlieb, A.I. 2003. Towards understanding acute destabilization of vulnerable atherosclerotic plaques. Cardiovasc. Pathol. 12:237-248.

10. Libby, P., et al. 1996. Macrophages and atherosclerotic plaque stability. Curr. Opin. Lipidol. 7:330-335.

11. Chyu, K.Y., and Shah, P.K. 2001. The role of inflammation in plaque disruption and thrombosis. Rev. Cardiovasc. Med. 2:82-91.

12. Shah, P.K. 1998. Role of inflammation and metalloproteinases in plaque disruption and thrombosis. Vasc. Med. 3:199-206.

13. Shah, P.K., et al. 1995. Human monocyte-derived macrophages induce collagen breakdown in fibrous caps of atherosclerotic plaques. Potential role of matrix-degrading metalloproteinases and implications for plaque rupture. Circulation. 92:1565-1569.

14. Mach, F., Schonbeck, U., and Libby, P. 1998. CD40 signaling in vascular cells: a key role in atherosclerosis? Atherosclerosis. 137(Suppl.):S89-S95.

15. Johnston, B., and Butcher, E.C. 2002. Chemokines in rapid leukocyte adhesion triggering and migration. Semin. Immunol. 14:83-92.

16. Vestweber, D., and Blanks, J.E. 1999. Mechanisms that regulate the function of the selectins and their ligands. Physiol. Rev. 79:181-213.

17. Konstantopoulos, K., Kukreti, S., and McIntire, L.V. 1998. Biomechanics of cell interactions in shear fields. Adv. Drug Deliv. Rev. 33:141-164.

18. Laudanna, C., Kim, J.Y., Constantin, G., and Butcher, E. 2002. Rapid leukocyte integrin activation by chemokines. Immunol. Rev. 186:37-46.

19. McEver, R.P., and Cummings, R.D. 1997. Role of
PSGL-1 binding to selectins in leukocyte recruitment. J. Clin. Invest. 100:485-491.

20. Chan, J.R., Hyduk, S.J., and Cybulsky, M.I. 2001. Chemoattractants induce a rapid and transient upregulation of monocyte alpha4 integrin affinity for vascular cell adhesion molecule 1 which mediates arrest: an early step in the process of emigration. J. Exp. Med. 193:1149-1158.

21. Sugihara-Seki, M. 2000. Flow around cells adhered to a microvessel wall. I. Fluid stresses and forces acting on the cells. Biorheology. 37:341-359.

22. Gaver, D.P., 3rd, and Kute, S.M. 1998. A theoretical model study of the influence of fluid stresses on a cell adhering to a microchannel wall. Biophys. J. 75:721-733.

23. Mohan, S., Mohan, N., Valente, A.J., and Sprague, E.A. 1999. Regulation of low shear flow-induced HAEC VCAM-1 expression and monocyte adhesion. Am. J. Physiol. 276:C1100-C1107.

24. Chappell, D.C., Varner, S.E., Nerem, R.M., Medford, R.M., and Alexander, R.W. 1998. Oscillatory shear stress stimulates adhesion molecule expression in cultured human endothelium. Circ. Res. 82:532-539.

25. Weber, C. 2003. Novel mechanistic concepts for the control of leukocyte transmigration: specialization of integrins, chemokines, and junctional molecules. J. Mol. Med. 81:4-19.

26. Charo, I.F., and Taubman, M.B. 2004. Chemokines in the pathogenesis of vascular disease. Circ. Res. 95:858-866.

27. Shyy, Y.J., Hsieh, H.J., Usami, S., and Chien, S. 1994. Fluid shear stress induces a biphasic response of human monocyte chemotactic protein 1 gene expression in vascular endothelium. Proc. Natl. Acad. Sci. U. S. A. 91:4678-4682.

28. Bao, X., Lu, C., and Frangos, J.A. 1999. Temporal gradient in shear but not steady shear stress induces PDGF-A and MCP-1 expression in endothelial cells: role of NO, NF kappa B, and egr-1. Arterioscler. Thromb. Vasc. Biol. 19:996-1003.

29. Kato, H., Uchimura, I., Nawa, C., Kawakami, A., and Numano, F. 2001. Fluid shear stress suppresses interleukin 8 production by vascular endothelial cells. Biorheology. 38:347-353.

30. Cheng, C., et al. 2004. The role of shear stress in atherosclerosis: action through gene expression and inflammation? Cell Biochem. Biophys. 41:279-294.

31. Weber, C., Schober, A., and Zernecke, A. 2004. Chemokines: key regulators of mononuclear cell recruitment in atherosclerotic vascular disease. Arterioscler. Thromb. Vasc. Biol. 24:1997-2008.

32. Whitman, S.C., Rateri, D.L., Szilvassy, S.J., Yokoyama, W., and Daugherty, A. 2004. Depletion of natural killer cell function decreases atherosclerosis in low-density lipoprotein receptor null mice. Arterioscler. Thromb. Vasc. Biol. 24:1049-1054.

33. Burke, A.P., et al. 2002. Traditional risk factors and the incidence of sudden coronary death with and without coronary thrombosis in blacks. Circulation. 105:419-424.

34. Farb, A., et al. 1996. Coronary plaque erosion without rupture into a lipid core. A frequent cause of coronary thrombosis in sudden coronary death. Circulation. 93:1354-1363.

35. Takeya, M., Yoshimura, T., Leonard, E.J., and Takahashi, K. 1993. Detection of monocyte chemoattractant protein-1 in human atherosclerotic lesions by an anti-monocyte chemoattractant protein-1 monoclonal antibody. Hum. Pathol. 24:534-539.

36. Rayner, K., Van Eersel, S., Groot, P.H., and Reape, T.J. 2000. Localisation of mRNA for JE/MCP-1 and its receptor CCR2 in atherosclerotic lesions of the ApoE knockout mouse. J. Vasc. Res. 37:93-102.

37. Guo, J., et al. 2005. Repopulation of apolipoprotein E knockout mice with CCR2-deficient bone marrow progenitor cells does not inhibit ongoing atherosclerotic lesion development. Arterioscler. Thromb. Vasc. Biol. 25:1014-1019.

38. Taub, D.D., Longo, D.L., and Murphy, W.J. 1996. Human interferon-inducible protein-10 induces mononuclear cell infiltration in mice and promotes the migration of human T lymphocytes into the peripheral tissues and human peripheral blood lymphocytes-SCID mice. Blood. 87:1423-1431.

39. Loetscher, M., et al. 1996. Chemokine receptor specific for IP10 and mig: structure, function, and expression in activated T-lymphocytes. J. Exp. Med. 184:963-969.

40. Luster, A.D., and Ravetch, J.V. 1987. Biochemical characterization of a gamma interferon-inducible cytokine (IP-10). J. Exp. Med. 166:1084-1097.

41. Libby, P., Schoenbeck, U., Mach, F., Selwyn, A.P., and Ganz, P. 1998. Current concepts in cardiovascular pathology: the role of LDL cholesterol in plaque rupture and stabilization. Am. J. Med. 104:14S-18S.

42. Kawamura, A., et al. 2003. CXCR3 chemokine receptor-plasma IP10 interaction in patients with coronary artery disease. Circ. J. 67:851-854.

43. Boisvert, W.A., Santiago, R., Curtiss, L.K., and Terkeltaub, R.A. 1998. A leukocyte homologue of the IL-8 receptor CXCR-2 mediates the accumulation of macrophages in atherosclerotic lesions of LDL receptor-deficient mice. J. Clin. Invest. 101:353-363.

44. Mizoue, L.S., Bazan, J.F., Johnson, E.C., and Handel, T.M. 1999. Solution structure and dynamics of the $\mathrm{CX} 3 \mathrm{C}$ chemokine domain of fractalkine and its interaction with an $\mathrm{N}$-terminal fragment of CX3CR1. Biochemistry. 38:1402-1414.

45. Lucas, A.D., et al. 2003. Smooth muscle cells in human atherosclerotic plaques express the fractalkine receptor CX3CR1 and undergo chemotaxis to the CX3C chemokine fractalkine (CX3CL1). Circulation. 108:2498-2504.

46. Ludwig, A., Berkhout, T., Moores, K., Groot, P., and Chapman, G. 2002. Fractalkine is expressed by smooth muscle cells in response to IFN-gamma and TNF-alpha and is modulated by metalloproteinase activity. J. Immunol. 168:604-612.

47. Hatakeyama, M., et al. 2004. Heparin inhibits IFNgamma-induced fractalkine/CX3CL1 expression in human endothelial cells. Inflammation. 28:7-13.

48. Lesnik, P., Haskell, C.A., and Charo, I.F. 2003. Decreased atherosclerosis in CX3CR $1^{-/-}$mice reveals a role for fractalkine in atherogenesis. J. Clin. Invest. 111:333-340. doi:10.1172/JCI200315555.

49. Combadiere, C., et al. 2003. Decreased atherosclerotic lesion formation in CX3CR1/apolipoprotein E double knockout mice. Circulation. 107:1009-1016.

50. Teupser, D., et al. 2004. Major reduction of atherosclerosis in fractalkine (CX3CL1)-deficient mice is at the brachiocephalic artery, not the aortic root. Proc. Natl. Acad. Sci. U. S. A. 101:17795-17800.

51. Gray, J.L., and Shankar, R. 1995. Down regulation of CD11b and CD18 expression in atherosclerotic 
lesion-derived macrophages. Am. Surg. 61:674-679; discussion 679-680.

52. de Bruijn, M.F., et al. 1996. High-level expression of the ER-MP58 antigen on mouse bone marrow hematopoietic progenitor cells marks commitment to the myeloid lineage. Eur. J. Immunol. 26:2850-2858.

53. Atkinson, J.B., Harlan, C.W., Harlan, G.C., and Virmani, R. 1994. The association of mast cells and atherosclerosis: a morphologic study of early atherosclerotic lesions in young people. Hum. Pathol. 25:154-159.

54. Schafer, A., et al. 2004. Novel role of the membrane- bound chemokine fractalkine in platelet activation and adhesion. Blood. 103:407-412.

55. Galli, S.J. 2000. Mast cells and basophils. Curr. Opin. Hematol. 7:32-39.

56. Laine, P., Naukkarinen, A., Heikkila, L., Penttila, A., and Kovanen, P.T. 2000. Adventitial mast cells connect with sensory nerve fibers in atherosclerotic coronary arteries. Circulation. 101:1665-1669.

57. Papadopoulos, E.J., et al. 2000. Mast cells migrate, but do not degranulate, in response to fractalkine, a membrane-bound chemokine expressed constitutively in diverse cells of the skin. Eur. J. Immunol.
30:2355-2361.

58. El-Shazly, A., et al. 2006. Fractalkine produced by airway smooth muscle cells contributes to mast cell recruitment in asthma. J. Immunol. 176:1860-1868.

59. Moll, P.R., Duschl, J., and Richter, K. 2004. Optimized RNA amplification using T7-RNA-polymerase based in vitro transcription. Anal. Biochem. 334:164-174.

60. von der Thusen, J.H., et al. 2002. Induction of atherosclerotic plaque rupture in apolipoprotein E-/- mice after adenovirus-mediated transfer of p53. Circulation. 105:2064-2070. 